\title{
ON QUASI-PURITY OF THE BRANCH LOCUS
}

\author{
ALEXANDER SCHMIDT
}

The notion of ramification is classical and important in arithmetic geometry. There are essentially two different approaches: the valuation theoretic notion of ramification and the scheme (or ring) theoretic one. In case of an extension of Dedekind domains, both notions coincide since regular local rings of dimension one are exactly the valuation rings of discrete rank one valuations. In higher dimension or without regularity assumptions the two notions of ramification diverge.

Let $K / k$ be a finitely generated field extension. A $k$-valuation of $K$ is a valuation $v$ on $K$ which is trivial on $k$. We call a normal, connected scheme $X / k$ separated and of finite type with function field $K$ a model of $K$. The normalization of $X$ in a finite, separable extension $L / K$ is denoted by $X_{L}$. The main result of this paper is the following

Theorem A (Quasi-purity of the branch locus). Let $L / K$ be a finite separable extension which is ramified at some $k$-valuation $w$ of $L$. Then there exists a model $X$ of $K$ and a (Weil) prime divisor $D \subset X_{L}$ which is ramified in the scheme morphism $X_{L} \rightarrow X$.

Assuming the existence of a regular, proper model $X$ of $K$, Theorem $\mathrm{A}$ is a straight-forward consequence of the Zariski-Nagata theorem on the purity of the branch locus. The existence of a regular, proper model of $K$ is known if $\operatorname{char}(k)=0$ [Hi64; if $\operatorname{char}(k)=p>0$ and $\operatorname{tr} \cdot \operatorname{deg}_{k} K \leq 2$ [Li78], and if $\operatorname{char}(k)=$ $p>0,\left[k: k^{p}\right]<\infty$ and tr. $\operatorname{deg}_{k} K=3$ [CP08, CP09]. In this paper we avoid assumptions on resolution of singularities by using M. Temkin's inseparable local uniformization theorem [Te13] instead.

As an application of Theorem $\mathrm{A}$ we show the following Theorem B, see [KS10] for the notion of curve-tameness and [Hü18] for that of tameness for étale morphisms of adic spaces. We recall the relevant definitions in Sections 4 and 5 .

Theorem B. Let $k$ be a field of positive characteristic, $X$ and $Y$ schemes, separated and of finite type over $k$ and $f: Y \rightarrow X$ a finite, étale $k$-morphism. Let

$$
\operatorname{Spa}(f): \operatorname{Spa}(Y, k) \longrightarrow \operatorname{Spa}(X, k)
$$

be the associated étale morphism of adic spaces.

Then $f$ is curve-tame if and only if $\operatorname{Spa}(f)$ is tame.

Date: November 29, 2018. 


\section{Passage to the algebraic closure}

Let $K$ be a field (imperfect, otherwise the following discussion is void), $\bar{K}$ an algebraic closure of $K, K^{s}$ the separable closure of $K$ in $\bar{K}$ and $K_{\infty}=K^{1 / p^{\infty}}$ the perfect closure of $K$ in $\bar{K}$. Then the natural map $\operatorname{Aut}(\bar{K} / K) \rightarrow \operatorname{Aut}\left(K^{s} / K\right)$, $\left.\sigma \mapsto \sigma\right|_{K^{s}}$ induces an isomorphism

$$
\varphi: \operatorname{Gal}\left(\bar{K} / K_{\infty}\right) \stackrel{\sim}{\longrightarrow} \operatorname{Gal}\left(K^{s} / K\right) .
$$

Lemma 1.1. Let $v$ be a valuation on $\bar{K}$ and let $v^{s}$ be its restriction to $K^{s}$. Then $\varphi$ induces isomorphisms

$$
D_{v} \stackrel{\sim}{\rightarrow} D_{v^{s}}, I_{v} \stackrel{\sim}{\rightarrow} I_{v^{s}}, \quad R_{v} \stackrel{\sim}{\rightarrow} R_{v^{s}},
$$

where the letters $D, I$ and $R$ denote the decomposition, inertia and ramification groups of the respective valuations.

Proof. Since $v$ is the only extension of $v^{s}$ to $\bar{K}$ [EP05, Corollary 3.2.10], we have for $\sigma \in \operatorname{Aut}(\bar{K} / K)$ that

$$
\sigma v=\left.v \Leftrightarrow \sigma\right|_{K^{s}}\left(v^{s}\right)=v^{s},
$$

hence $\varphi$ induces an isomorphism $D_{v} \stackrel{\sim}{\rightarrow} D_{v^{s}}$.

Since $K_{\infty} / K$ is purely inseparable, the same is true for the residue field extension

$$
\kappa\left(\left.v\right|_{K_{\infty}}\right) / \kappa\left(\left.v\right|_{K}\right) .
$$

Hence we obtain the commutative diagram

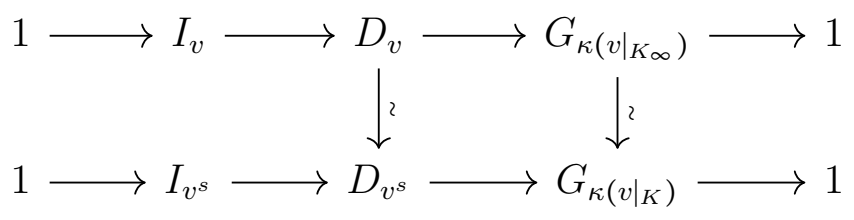

inducing the claimed isomorphism $I_{v} \stackrel{\sim}{\rightarrow} I_{v^{s}}$. Finally, the ramification groups are the $p$-Sylow subgroups of the inertia groups, showing that also $R_{v} \stackrel{\sim}{\rightarrow} R_{v^{s}}$.

The ramification indices are in general not preserved under inseparable base change:

Example 1.2. Let $K=\mathbb{F}_{p}(X, Y)$ and $L=K[T] /\left(T^{p}-X T-Y\right)$. The valuation of $K$ associated with $X$ does not split in $L / K$, the ramification index is equal to 1 , and the residue field extension is the inseparable extension $\mathbb{F}_{p}\left(Y^{1 / p}\right) / \mathbb{F}_{p}(Y)$. Now consider $K^{\prime}=K\left(Y^{1 / p}\right)=\mathbb{F}_{p}\left(X, Y^{1 / p}\right), L^{\prime}=K^{\prime} L$. Over $K^{\prime}$ the polynomial $T^{p}-X T-Y$ can be written as $\left(T-Y^{1 / p}\right)^{p}-X\left(T-Y^{1 / p}\right)+X Y^{1 / p}$. Up to a substitution, it is an Eisenstein polynomial. Hence the valuation of $K^{\prime}$ associated with $X$ does not split in $L^{\prime} / K^{\prime}$, the ramification index is equal to $p$, and the residue extension is trivial. 


\section{2. ÉtALE VERSUS UNRAMIFIED}

The following lemma seems to be well-known but we could not find a reference.

Lemma 2.1. Let $L / K$ be a finite field extension, $w$ a non-archimedean valuation of $L$ and $v=\left.w\right|_{K}$. Then $w / v$ is unramified (in the valuation-theoretic sense) if and only if $\mathcal{O}_{v} \rightarrow O_{w}$ is étale in the ring-theoretic sense.

Proof. Let $\mathcal{O}_{v, L}$ be the integral closure of $\mathcal{O}_{v}$ in $L$. Then, for every maximal ideal $\mathfrak{m} \subset \mathcal{O}_{v, L}$, the localization $\left(\mathcal{O}_{v, L}\right)_{\mathfrak{m}}$ is a valuation ring of a valuation of $L$ which extends $v$ and, by [EP05, Theorem 3.2.13], the assignment

$$
\mathfrak{m} \longmapsto\left(\mathcal{O}_{v, L}\right)_{\mathfrak{m}}
$$

gives a 1:1-correspondence between the maximal ideals of $\mathcal{O}_{v, L}$ and those valuations. In particular, $\mathcal{O}_{v, L}$ is semi-local. As is well known, the inertia group of the action of $G=\operatorname{Gal}(L / K)$ detects ramification in the valuation-theoretic sense. The same is true for étale by [Ra70, Ch. X, Theorem 1].

Example 2.2 (A. Holschbach). Consider the field $K=\mathbb{C}((T))$ and let $L=$ $K\left(T^{\frac{1}{2}}\right)$. Consider

$$
K_{\infty}=\bigcup_{r=1}^{\infty} K\left(T^{1 / 3^{r}}\right), L_{\infty}=L K_{\infty}
$$

Then $L_{\infty} / K_{\infty}$ is ramified in the valuation-theoretic sense. The associated rings of integers are

$$
A=\mathbb{C}[[T]]\left[T^{1 / 3^{r}}, r \geq 1\right], \quad B=\mathbb{C}[[T]]\left[T^{1 / 2 \cdot 3^{r}}, r \geq 1\right] .
$$

Hence the ring extension $B / A$ satisfies $\mathfrak{m}_{A} B=\mathfrak{m}_{B}$. But it is not of finite type, hence not étale in the ring-theoretic sense.

Lemma 2.3. Let $k$ be a field, $X$ a normal, connected and separated scheme of finite type over $k, K=k(X), L / K$ a finite, separable field extension and $Y$ the normalization of $X$ in $L$. Let $w$ be a $k$-valuation on $L$ having center $y \in Y$ and let $v$ be the restriction of $w$ to $K$. Assume that $Y \rightarrow X$ is étale at $y$. Then $w / v$ is unramified.

Proof. Let $s$ be the special point of $\operatorname{Spec}\left(\mathcal{O}_{w}\right)$ and $t$ its image under $\operatorname{Spec}\left(\mathcal{O}_{w}\right) \rightarrow$ $Y \times_{X} \operatorname{Spec}\left(\mathcal{O}_{v}\right)$. Since $t$ maps to $y$ in $Y$ and $Y \rightarrow X$ is étale at $y$, the base change $Y \times_{X} \operatorname{Spec}\left(\mathcal{O}_{v}\right) \rightarrow \operatorname{Spec}\left(\mathcal{O}_{v}\right)$ is étale at $t$. As étale schemes over normal schemes are normal [Ra70, Ch. VII, Prop. 2], the local ring of $Y \times_{X} \operatorname{Spec}\left(\mathcal{O}_{v}\right)$ at $t$ is normal and hence isomorphic to $\mathcal{O}_{w}$ (cf. the proof of Lemma 2.1). Since $Y \times_{X} \operatorname{Spec}\left(\mathcal{O}_{v}\right) \rightarrow \operatorname{Spec}\left(\mathcal{O}_{v}\right)$ is étale at $t, \mathcal{O}_{w} / \mathcal{O}_{v}$ is étale.

\section{QUASI-PURITY}

Let $K / k$ be a finitely generated field extension of transcendence degree $d$ and let $v$ be a discrete rank one $k$-valuation on $K$. By Abhyankar's inequality (cf., 
e.g., [KS10, Prop. 3.2]), we have for the residue field $K v$ of $v$ that

$$
\text { deg. } \operatorname{tr}_{k}(K v) \leq d-1 \text {. }
$$

We call $v$ geometric, if equality holds in $(*)$. By [Li02, §8, Thm. 3.26(b)] $v$ is geometric if and only if there exists a model $X$ of $K$ and a Weil prime divisor $D \subset X$ with $\mathcal{O}_{v}=\mathcal{O}_{X, D}$. Hence Theorem $\mathrm{A}$ is equivalent to

Theorem 3.1. Let $k$ be a field, $K / k$ a finitely generated field extension and $L / K$ a finite, separable extension. Assume there exists a $k$-valuation $w$ on $L$ with restriction $v$ to $K$ such that $w / v$ is ramified. Then there exists a geometric discrete rank one $k$-valuation $W$ on $L$ with restriction $V$ to $K$ such that $W / V$ is ramified.

Proof. We choose a proper model $X$ of $K$. By M. Temkin's inseparable local uniformization theorem [Te13, Cor. 1.3.3], we find a regular, connected $k$-scheme $X^{\prime}$ and a $k$-morphism $X^{\prime} \rightarrow X$ such that $K^{\prime}=k\left(X^{\prime}\right) / K=k(X)$ is a finite, purely inseparable extension and the unique $k$-valuation $v^{\prime}$ of $K^{\prime}$ extending $v$ has center in $X^{\prime}$. Let $L^{\prime}=L K^{\prime}$ be the composite (in some algebraic closure of $K$ ) and $w^{\prime}$ the unique $k$-valuation of $L^{\prime}$ lying over $w$. Then $\left.w^{\prime}\right|_{K^{\prime}}=v^{\prime}$ and $w^{\prime} / v^{\prime}$ is ramified by Lemma 1.1. Hence, by Lemma 2.3, the scheme morphism $X_{L}^{\prime} \rightarrow X^{\prime}$ is not étale. By Zariski-Nagata purity [SGA2, X, Théorème 3.4], we find a ramified divisor, hence a geometric rank one $k$-valuation $W^{\prime}$ of $L^{\prime}$ with restriction $V^{\prime}$ to $K^{\prime}$ such that $W^{\prime} / V^{\prime}$ is ramified. Denoting the respective restrictions to $L$ and $K$ by $W$ and $V$, another application of Lemma 1.1 shows that $W / V$ is ramified.

We will use the following mild sharpening of Theorem $\mathrm{A}$ in the proof of Theorem 4.4 below.

Corollary 3.2. Under the assumptions of Theorem $\triangle$ let $U$ be a model of $K$ such that $U_{L} \rightarrow U$ is étale. Then we can choose the model $X$ of $K$ asserted in Theorem $A$ in such a way that there is an open $k$-immersion $j: U \hookrightarrow X$ and the ramified prime divisor $D$ is contained in $X_{L} \backslash U_{L}$.

Proof. We choose a proper model $\bar{U}$ of $K$ containing $U$ as an open subscheme. By Theorem 3.1, we find a geometric discrete rank one $k$-valuation $W$ of $L$ with restriction $V$ to $K$ such that $W / V$ is ramified. Since $\bar{U}$ is proper, $V$ has a nonempty center on $\bar{U}$ which is contained in $\bar{U} \backslash U$ since $U_{L} \rightarrow U$ is étale. Following [Li02, §8, Exercise 3.14], by successively blowing up $\bar{U}$ in centers contained in $\bar{U} \backslash U$ and finally normalizing, we find a normal compactification $X$ of $U$ such that $V$ is the valuation associated to a point $x \in X \backslash U$ of codimension one in $X$. This finishes the proof.

\section{Curve-tameness}

Let $k$ be a field of positive characteristic. By variety we mean a separated scheme of finite type over $k$, a curve $C$ is an integral variety with $\operatorname{dim} C=1$ and 
by étale covering we mean finite, étale morphism. For a regular curve $C$ there exists a unique regular curve $P(C)$ which is proper and contains $C$ as a dense open subscheme. Recall that an étale covering $C^{\prime} \rightarrow C$ of regular curves is called tamely ramified along $P(C) \backslash C$ if for every $x \in P(C) \backslash C$ the associated valuation $v_{x}$ is tamely ramified in the finite, separable field extension $k\left(C^{\prime}\right) / k(C)$. This definition extends to the case of general regular varieties of dimension one by requiring tameness on every connected component.

Recall the following definitions from [KS10]:

Definition 4.1. An étale covering $Y \rightarrow X$ of varieties is curve-tame if for any morphism $C \rightarrow X$ with $C$ a regular curve, the base change $Y \times{ }_{X} C \rightarrow C$ is tamely ramified along $P(C) \backslash C$.

If, in addition, $X$ and $Y$ are normal and connected, we say that $Y \rightarrow X$ is valuation-tame if every $k$-valuation of $k(X)$ is tamely ramified in the field extension $k(Y) / k(X)$. This definition extends to coverings of general normal varieties by requiring valuation tameness on every connected component.

By definition, the notions of curve- and valuation-tameness agree for coverings of regular curves. The statement of the next lemma follows directly from the definitions.

Lemma 4.2. Let $g: Z \rightarrow Y$ and $f: Y \rightarrow X$ be étale coverings. If $g$ and $f$ are curve-tame, then the same holds for $f \circ g$. If $f \circ g$ is curve-tame, then $g$ is curve-tame and if, in addition, $g$ is surjective, then also $f$ is curve-tame.

The same holds for valuation-tame instead of curve-tame.

Lemma 4.3. (i) An étale covering of connected varieties is curve-tame if and only if its Galois closure is curve-tame.

(ii) An étale covering of normal, connected varieties is valuation-tame if and only if its Galois closure is valuation-tame.

Proof. If the Galois closure $\tilde{Y} \rightarrow X$ of $Y \rightarrow X$ is curve-tame, then $Y \rightarrow X$ is curvetame by Lemma 4.2. Directly from the definition we see that for curve-tame coverings $Y_{1} \rightarrow X, Y_{2} \rightarrow X$ also their fibre product $Y_{1} \times_{X} Y_{2} \rightarrow X$ is curve-tame. Now the Galois closure $\widetilde{Y} \rightarrow X$ of $Y \rightarrow X$ occurs as a connected component (of maximal degree) of the $d$-fold self product $Y \times_{X} \cdots \times_{X} Y$ with $d=\operatorname{deg}(Y / X)$. Hence $\widetilde{Y} \rightarrow X$ is curve-tame if $Y \rightarrow X$ is.

The same arguments apply for valuation-tameness.

The main result of this section is

Theorem 4.4. Let $k$ be a field of positive characteristic and let $f: Y \rightarrow X$ be a finite, étale morphism of regular $k$-varieties. Then $f$ is curve-tame if and only if it is valuation-tame. 
Remark 4.5. Theorem 4.4 sharpens [KS10, Theorem 4.4] (which makes an assumption on the existence of regular, proper models) in the case that the base scheme is the spectrum of a field.

Proof of Theorem 4.4. By Lemma 4.3, we may assume that $f: Y \rightarrow X$ is a Galois covering of connected varieties.

Assume that there exists a $k$-valuation $w$ of $k(Y)$ which is wildly ramified in $k(Y) / k(X)$. Let $1 \neq R_{w} \subset \operatorname{Gal}(Y / X)$ be the ramification group of $w$ and let $G \subset R_{w}$ be a cyclic subgroup of order $p$. Setting $Z=Y / G$, we obtain a cyclic Galois covering $Y / Z$ of order $p$ in which $w$ is (wildly) ramified. By Corollary 3.2 , we find a normal, connected variety $\bar{Z}$ containing $Z$ as a dense open subscheme and a prime divisor $D \subset \bar{Z}$ which ramifies in $\bar{Z}_{k(Y)} \rightarrow \bar{Z}$. By the Key Lemma 2.4 of [KS10], we find a regular curve $C$ and a morphism $C \rightarrow Z$ such that the base change $Y \times_{Z} C \rightarrow C$ is (wildly) ramified along $P(C)$. Hence $Y \rightarrow Z$ is not curve-tame and by Lemma 4.2, also $Y \rightarrow X$ is not curve-tame. This shows that curve-tameness implies valuation tameness.

The other implication is part of [KS10, Theorem 4.4].

\section{TAME MORPHISMS OF ADIC SPACES}

We refer the reader to [Hu96] for basic notions on adic spaces. Following [Hü18, $\S 3]$, we call an étale morphism $\mathscr{Y} \rightarrow \mathscr{X}$ of adic spaces tame if for every point $y \in \mathscr{Y}$ with image $x \in \mathscr{X}$ the extension of the valuations associated to $y$ and $x$ is (at most) tamely ramified.

In [Te11] M. Temkin associates with a morphism of schemes $X \rightarrow S$ a discretely ringed adic space $\operatorname{Spa}(X, S)$. If $X=\operatorname{Spec}(A)$ and $S=\operatorname{Spec}(R)$ are affine, then $\operatorname{Spa}(X, S)$ coincides with Huber's affinoid adic space $\operatorname{Spa}\left(A, A^{+}\right)$, where $A$ is endowed with the discrete topology and $A^{+}$is the integral closure of the image of $R$ in $A$.

If $S=\operatorname{Spec}(k)$ is the spectrum of a field and $X$ is a variety over $k$, then the underlying set of points of $\operatorname{Spa}(X, k)$ is the following:

$$
|\operatorname{Spa}(X, k)|=\coprod_{x \in X} \operatorname{Val}_{k}(k(x)) \quad \text { (set-theoretically). }
$$

Here $\operatorname{Val}_{k}(k(x))$ is the set of $k$-valuations of $k(x)$.

An étale morphism of $k$-varieties $Y \rightarrow X$ induces an étale morphism of adic spaces $\mathrm{Spa}(Y, k) \rightarrow \mathrm{Spa}(X, k)$, cf. [Hu96]. With this preparations we are ready to prove Theorem $\mathrm{B}$,

Proof of Theorem $B$. By definition, $f$ is curve-tame if and only if for every point $y \in Y_{1}$ (i.e. the closure of $y$ is a curve) with image $x \in X$, the extension $k(y) / k(x)$ is tamely ramified at every $v \in \operatorname{Val}_{k} k(y)$. In other words, the tameness of $\operatorname{Spa}(f)$ trivially implies the curve-tameness of $f$. 
Conversely, assume that $f$ is curve-tame. Let $y \in Y$ be a point with image $x \in X$ and $v \in \operatorname{Val}_{k} k(y)$. We have to show that $v$ is tamely ramified in $k(y) / k(x)$. Replacing $X$ by the closure of $x$ and using that curve-tameness is stable under base change, we may assume that $x$ is the generic point of the integral variety $X$. Furthermore, after replacing $X$ by an open subscheme, we may assume that $X$ (and hence also $Y$ ) is regular. Then, by Theorem 4.4, $f$ is valuation-tame, hence $v$ is tamely ramified in $k(y) / k(x)$.

\section{ACKNOWLEDGEMENT}

The author thanks A. Holschbach for helpful discussions and for providing Example 2.2. Moreover, we thank K. Hübner for her comments on a preliminary version of this article.

\section{REFERENCES}

[CP08] Cossart, V.; Piltant, O. Resolution of singularities of threefolds in positive characteristic. I. Reduction to local uniformization on Artin-Schreier and purely inseparable coverings. J. Algebra 320 (2008), no. 3, 1051-1082.

[CP09] Cossart, V.; Piltant, O. Resolution of singularities of threefolds in positive characteristic. II. J. Algebra 321 (2009), no. 7, 1836-1976.

[EP05] Engler, A. J.; Prestel, A. Valued fields. Springer Monographs in Mathematics. SpringerVerlag, Berlin, 2005.

[Hi64] Hironaka, H. Resolution of singularities of an algebraic variety over a field of characteristic zero. I, II. Ann. of Math. (2) 79 (1964), 109-203; ibid. (2) 791964 205-326.

[Hu96] Huber,R. Étale cohomology of rigid analytic varieties and adic spaces. Aspects of Mathematics. Vieweg, 1996.

[Hü18] Hübner, K. The adic tame site. arXiv:1801.04776 [math.AG]

[KS10] Kerz, M.; Schmidt, A. On different notions of tameness in arithmetic geometry. Math. Ann. 346 (2010), no. 3, 641-668.

[Li78] Lipman, J. Desingularization of two-dimensional schemes. Ann. of Math. 107 (1978) 151-207.

[Li02] Liu, Q. Algebraic geometry and arithmetic curves. Oxford Graduate Texts in Mathematics, 6. Oxford Science Publications. Oxford University Press, Oxford, 2002.

[SGA2] Grothendieck, A. Cohomologie locale des faisceaux cohérents et théorèmes de Lefschetz locaux et globaux (SGA 2). Advanced Studies in Pure Math., Vol. 2. North-Holland Publishing Co., Amsterdam; Masson \& Cie, Éditeur, Paris, 1968.

[Ra70] Raynaud, M. Anneaux locaux henséliens. Lecture Notes in Mathematics, Vol. 169 Springer-Verlag, Berlin-New York 1970

[Te11] Temkin, M. Relative Zariski-Riemann spaces. Israel J. Math. 185 (2011), 1-42.

[Te13] Temkin, M. Inseparable local uniformization. J. Algebra 373 (2013), 65-119.

Mathematisches Institut, Universität Heidelberg, Im Neuenheimer Feld 205, 69120 Heidelberg, Deutschland

E-mail address: schmidt@mathi.uni-heidelberg.de 